Relations industrielles

Industrial Relations

\title{
Le comportement des salaires dans l'industrie de l'imprimerie de Montréal et du district
}

1940-1949

\section{Raymond Gaudreau}

Volume 5, numéro 10, juillet 1950

URI : https://id.erudit.org/iderudit/1023412ar

DOI : https://doi.org/10.7202/1023412ar

Aller au sommaire du numéro

Éditeur(s)

Département des relations industrielles de l’Université Laval

ISSN

0034-379X (imprimé)

1703-8138 (numérique)

Découvrir la revue

Citer cet article

Gaudreau, R. (1950). Le comportement des salaires dans l'industrie de

l'imprimerie de Montréal et du district : 1940-1949. Relations industrielles /

Industrial Relations, 5(10), 95-97. https://doi.org/10.7202/1023412ar

Tous droits réservés @ C Département des relations industrielles de l’Université Laval, 1950
Ce document est protégé par la loi sur le droit d'auteur. L'utilisation des services d'Érudit (y compris la reproduction) est assujettie à sa politique d'utilisation que vous pouvez consulter en ligne.

https://apropos.erudit.org/fr/usagers/politique-dutilisation/ 
D'ici quelque temps, surtout si une crise économique survient, d'autres établissements fermeront leurs portes. On continuera à dire, en certains milieux opposés à la convention extensionnée, que ces manufacturiers ont fermé à cause de la convention. En fait, avec ou sans convention, ces firmes fermeraient de la même manière; avec toutefois une différence que, sans convention collective extensionnée, elles fermeraient après avoir baissé leurs salaires à un état lamentable et entraîné une réduction semblable de salaires dans les ateliers concurrents.

Le décret de la chaussure a eu pour but de faire disparaître la concurrence sur le salaire et sur le gagne-pain de la famille ouvrière; les documents révélés plus haut semblent démontrer qu'il a obtenu ce résultat, sans pour cela mettre en péril la vie de l'industrie.

\title{
LE COMPORTEMENT DES SALAIRES DANS L'INDUSTRIE DE L'IMPRIMERIE DE MONTRÉAL ET DU DISTRICT
}

\author{
$1940-1949$ \\ RAYMOND GAUdREAU
}

Une des premières conventions collectives avec extension juridique, dans la province de Québec, fut celle de l'industrie de l'imprimerie de Montréal et du district, conclue en 1936. Depuis lors, de nouvelles conventions collectives se sont succédé d'année en année. Ces diverses conventions collectives couvrent les établissements d'imprimerie de Montréal et d'un territoire de cent milles de ses limites.

Tous les établissements d'imprimerie sis dans ce territoire se répartissent en trois zones, ainsi délimitées: Zone I: l'Ile de Montréal et le territoire jusqu'à dix milles de ses limites. Zone II: les municipalités suivantes et le territoire jusqu'à deux milles de leurs limites respectives: East Templeton, Granby, Hull, Joliette, St-Hyacinthe, St-Jean, Iberville, St-Jérôme, Sherbrooke, Sorel, Trois-Rivières et Valleyfield. Les établissements qui publiaient ou imprimaient un ou plusieurs journaux hebdomadaires au 15 juin, 1946, sont exclus de cette zone. Zone III: tout le territoire non compris dans les zones I et II. Les établissements sis dans les zones II et III qui publiaient ou imprimaient des journaux hebdomadaires au 15 juin, 1946, sont groupés sous le titre Zones 2a, 3a.

Ces précisions aident à mieux comprendre le comportement des salaires dans l'industrie de l'imprimerie. Elles situent le cadre de notre étude: Montréal et un territoire de 100 milles de ses limites. Par ailleurs, aux termes du décret donnant une extension juridique à la convention collective, les taux minima de salaires et le nombre d'heures régulières de travail sont fixés pour chaque zone. Notons immédiatement que le comportement général des salaires est affecté par ces taux minima de salaires. La rémunération du travailleur marginal étant automatiquement élevée par une hausse du taux minimum de salaires, le taux moyen payé subit par le fait même une «pression » vers la hausse.

A la lumière de ces données, étudions le comportement des salaires sous deux aspects: le volume de salaires payés et les taux minima et moyen de salaires.

\section{Volume de salaires payés}

Le volume des salaires est passé de près de 3 millions en 1940 à 11.5 millions en 1949 (voir tableau ci-après). Les salaires payés, de 1940 à 1949 , offrent un comportement quelque peu différent de celui des heures travaillées et du nombre moyen d'employés. Le contrôle fédéral des salaires durant la guerre a freiné l'expansion du volume des salaires payés. La décade 1940-1949 se divise par conséquent en deux périodes distinctes: de 1940 au ler semestre de 1945, et du 2e semestre de 1945 à 1949.

Entre 1940 et 1945, à une augmentation de $72 \%$ dans les heures travaillées et de $52 \%$ dans le nombre d'employés, correspond une augmentation de $97 \%$ dans le volume de salaires payés. Par contre, de 1945 à 1949, l'augmentation se fait au rythme suivant: heures travaillées, $17.9 \%$; nombre moyen d'employés, $26.0 \%$; gages payés, $96.1 \%$. En somme les salaires payés depuis 1945 ont continué d'augmenter à un rythme accéléré alors que les heures travaillées et le nombre moyen d'ouvriers se sont progressivement stabilisés.

Depuis 1940, le volume des salaires a augmenté de $286.6 \%$, comparé à une augmentation de $\mathbf{1 0 2 . 8 \%}$ dans les heures travaillées et de $\mathbf{9 2 . 1 \%}$ 
dans le nombre moyen d'ouvriers. Cette hausse considérable des salaires dans l'industrie de l'imprimerie est de beaucoup supérieure à l'augmentation du revenu des travailleurs canadiens en général, durant la même période. D'après les chiffres du Bureau fédéral de la statistique, le revenu total des travailleurs canadiens a augmenté de $162 \%$ entre 1940 et $1949 .\left({ }^{\circ}\right)$

\section{Taux horaires de salaires}

Pour demeurer dans le cadre de cet article, nous nous bornerons à considérer le mouvement des taux horaires de salaires de la zone I. Ce sont les plus représentatifs puisque $85 \%$ du volume des salaires sont payés dans la zone I. Les taux horaires de salaires des autres zones ont, d'ailleurs, suivi sensiblement le même comportement que ceux de la zone I depuis 1940.

Le taux minimum légal pour les compagnons est passé de $\$ 0.80$ à $\$ 1.40$ entre 1940 et 1949 , soit une augmentation de $75 \%$. Le taux de base moyen payé, durant la même période, est passé de $\$ 0.85$ à $\$ 1.55$, soit une augmentation de $87 \%$.

Le taux minimum légal, de 1940 à 1946, n’a changé qu'en fonction des deux bonis de vie chère autorisés par le gouvernement fédéral. Mais depuis 1946, c'est-à-dire depuis que le marché du travail est redevenu libre, six augmentations se sont produites dans le taux minimum. De $\$ 0.88$ qu'il était en 1946, il s'établit à $\$ 1.40$ en 1949 , soit une augmentation de $60 \%$. Ceci représente $80 \%$ de l'augmentation totale depuis dix ans. A remarquer également que l'amplitude des augmentations de taux minima de salaires de 1946 à 1949 va pratiquement toujours croissant: en 1946, $\$ 0.06$; en 1948, $\$ 0.16$; en 1949 , $\$ 0.30$.

De 1940 à 1945, le taux horaire moyen payé demeure à peu près aussi stationnaire que le taux minimum légal: $1940, \$ 0.83 ; 1944, \$ 0.95$. Mais à partir de 1945, la marge s'agrandit entre les deux taux, jusqu'en 1949 alors qu'elle revient au niveau de 1946. Ce phénomène peut s'expliquer ainsi: 1. l'industrie de l'imprimerie a subi la hausse générale des salaires qui a suivi la guerre et la fin du contrôle des salaires; 2. l'augmentation gigantesque dans la production a provoqué une augmentation parallèle dans la demande pour les produits d'imprimerie. A cause du caractère spécialisé de l'industrie de l'imprimerie et, d'autre part, de la rareté relative de main-d'oeuvre qualifiée, il y a eu plus d'heures régulières de travail, et, en proportion, plus d'heures supplémentaires. Ceci a

(*) Revue Statistique du Canada, mars 1950. sans doute contribué pour beaucoup à l'augmentation du taux moyen de base payé.

Les indices du coût de la vie, des taux minima et des taux moyens payés permettent plusieurs conclusions intéressantes. Il est nécessaire cependant de bien délımiter la portée des termes. L'indice du coût de la vie est celui préparé par le Bureau fédéral de la statistique. Cet indice vaut pour le Canada en général. Le taux minimum légal est le taux de salaire minimum qui doit être payé à un compagnon dans la zone I aux termes des décrets relatifs aux métiers de l'imprimerie de Montréal et du district. Le taux moyen payé est le taux de base moyen pour une heure régulière de travail.

Depuis 1939, trois phases caractérisent le comportement des taux de salaires comparé au coût de la vie. De 1939 à 1941, l'indice du coût de la vie et l'indice du taux horaire moyen se suivent de près. Puis vient la période de 1941 à 1944 durant laquelle, les salaires étant «gelés», l'indice du coût de la vie dépasse celui du taux de base moyen. Mais à partir de 1944, l'indice du taux de base moyen prend les devants. L'indice des salaires et l'indice du coût de la vie subissent une forte hausse à la fin de la guerre, soit durant les années 1946 et 1947. En 1948, la montée en spirale du coût de la vie est arrêtée. Par contre, les indices de taux de salaires (taux minimum et taux moyen) continuent de s'élever à un rythme de plus en plus accéléré.

Les chiffres suivants résument bien ce qui s'est passé depuis 10 ans:

\begin{tabular}{|c|c|c|c|}
\hline & $\begin{array}{c}\text { Indice du coût } \\
\text { de la vie }\end{array}$ & $\begin{array}{l}\text { Indice du taux } \\
\text { horaire légal } \\
\text { minimum }\end{array}$ & $\begin{array}{c}\text { Indice du } \\
\text { taux de base } \\
\text { moyen }\end{array}$ \\
\hline $\begin{array}{l}1940 \\
1945 \\
1949\end{array}$ & $\begin{array}{l}105.6 \\
119.5 \\
160.8\end{array}$ & $\begin{array}{l}106.7 \\
117.3 \\
186.7\end{array}$ & $\begin{array}{l}106.5 \\
124.8 \\
198.7\end{array}$ \\
\hline
\end{tabular}

Les ouvriers de l'industrie de l'imprimerie, ouvriers hautement spécialisés, sont donc rémunérés à un taux minimum et à un taux de base moyen plus que suffisants pour faire face au coût de la vie tel qu'estimé par le gouvernement fédéral.

L'augmentation considérable du volume des salaires de même que la hausse non moins considérable dans les taux horaires de salaires, notamment depuis la fin de la guerre, témoignent de la prospérité et du progrès considérable de l'industrie de l'imprimerie chez nous. Nous pouvons également conclure - cela est aujourd'hui un lieu commun mais il est utile de le rappeler - que le régime des conventions collectives à extension juridique et des comités paritaires crée, au sein de l'industrie, une atmosphère de paix et d'entente éminemment favorable aux ouvriers comme aux patrons. 


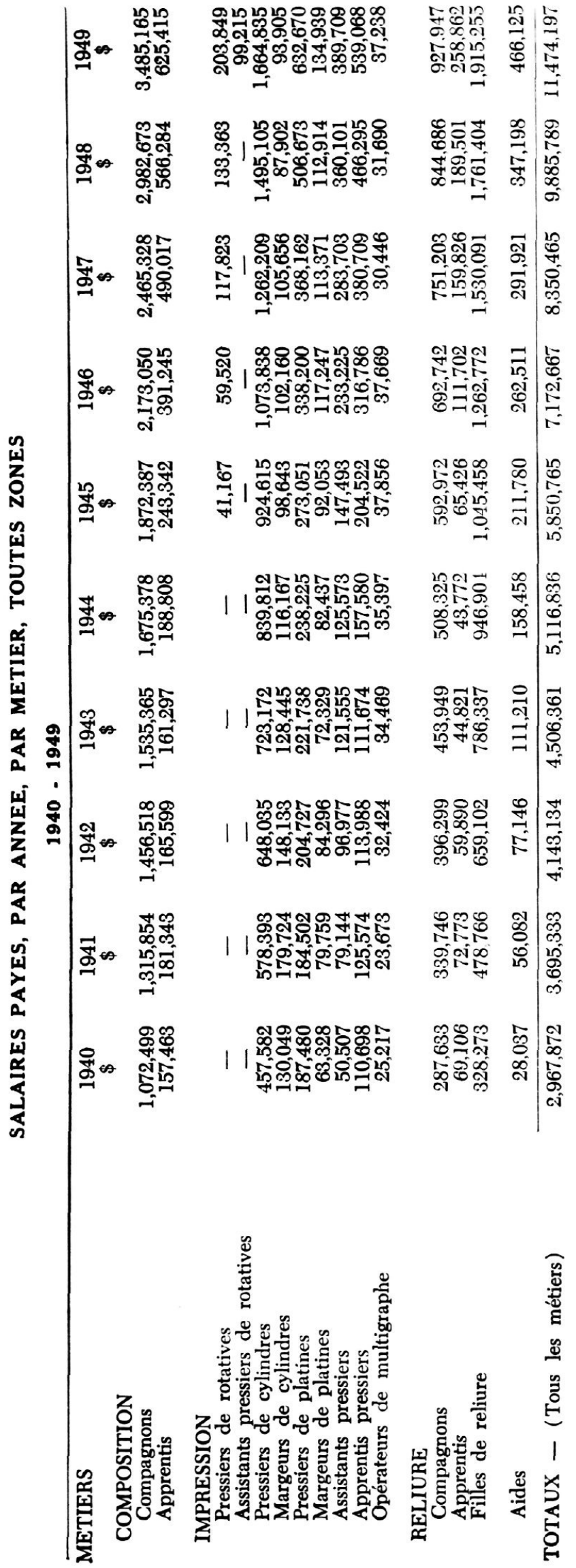

\section{JURISPRUDENCE DU TRAVAIL}

\section{Prescription et lois ouvrières}

\&e 17 août 1945, la demanderesse s'engagea comme institutrice pour les défendeurs, à raison de $\$ 400.00$ par année. Elle exécuta son contrat et reçut la somme de $\$ 400.00$. La Commission scolaire de St-Philippe est dans le district d'inspection no 60 . Les institutrices de ce district formèrent une association suivant la Loi des relations ouvrières et la Loi des différends entre les services publics et leurs salariés (S.R.Q. 1941, Ch. 162A et 169). L'association est munie d'un certificat en date du 2 août 1944 et ainsi est agent de négociation des institutrices catholiques au service des défendeurs. Les parties n'ayant pu négocier un projet de convention collective, le ministre ordonna la formation d'un conseil d'arbitrage afin de régler le différend. Le Conseil d'arbitrage rendit sa décision le 11 février 1946. La sentence arbitrale fut déposée entre les mains du ministre du travail et communiquée aux défendeurs. Elle constitue un titre en faveur de la demanderesse, tel que décidé par la Cour d'appel dans Association catholique des institutrices rurales du district no 60 vs Commissaires d'écoles de St-Pascal (1947 BR 728). La demanderesse, selon cette sentence, avait droit à la somme de $\$ 730.00$. Il lui est dû une solde de $\$ 330.00$ qu'elle réclame. $>$

Le débat est ramené à une question de prescription par la défense qui prétend, pour cette cause, que la sentence arbitrale est inopérante.

Or voici en substance le jugement. La Loi des relations ouvrières, la Loi des différends ouvriers de Québec et la Loi des différends entre les services publics et leurs salariés n'édictent aucune prescription de salaires. Par ailleurs, la Loi des syndicats professionnels, la Loi de la convention collective et la Loi du salaire minimum qui édictent une prescription de six mois, ne s'appliquent pas dans l'espèce. Il faut donc recourir à l'art. 2260, par. 6, C.C. Dès lors, la réclamation d'une institutrice pour le paiement de l'augmentation de son salaire fixée par une sentence arbitrale se prescrit par cinq ans, les parties admettant qu'il n'y a jamais eu de convention particulière.

(Mlle Gagnon vs Les commissaires d'écoles pour la municipalité de Saint-Philippe, Kamouraska, 24 janv. 1949; Président, Juge Langlais; avocat de la demanderesse, Me M.-L. Beaulieu, c.r.; avocat des défendeurs, Me V. Trépanier, c.r.; cf. R.J. de Q., C.S., 1949, p. 201.)

\section{Sécurité syndicale}

Dans un récent arbitrage, les membres du tribunal rejettent à l'unanimité une demande « d'atelier d'union * en invoquant les raisons suivantes: « La liberté individuelle pour laquelle les unions elles-mêmes se sont battues dans le passé, liberté individuelle qui est actuellement sanctionnée par le Traité des droits de d'homme, signé par notre pays et l'économie de nos lois, ne semble pas pouvoir nous autoriser à accorder cette demande $\$$.

(McLennan Lumber Limited, Montréal, et l'Association canadienne des travailleurs du bois, local no 16, Inc.; sentence unanime, le 18 juillet 1949; président, M. le juge Irénée Lagarde; arbitre patronal, Me Philippe Lamarre, c.r.; arbitre syndical, M. Léopold Lavoie.) 